\title{
Unusual complications in siblings with marfanoid phenotype
}

\author{
D G Harendra de Silva, T P J Gunawardena, F M K Law
}

\begin{abstract}
Marfan's syndrome has diverse manifestations that overlap with those seen in other connective tissue disorders. Visceral diverticula have been described only once in four adults with marfanoid features of recessive inheritance. Two siblings of a consanguineous marriage with marfanoid features, visceral diverticula, and diaphragmatic eventration are reported. (Arch Dis Child 1996;75:247-248)
\end{abstract}

Keywords: marfanoid features, visceral diverticula, diaphragmatic eventration.

In addition to the triad of skeletal, ocular, and cardiovascular abnormalities, the phenotypic features of Marfan's syndrome have expanded to include spontaneous pneumothorax with other congenital lung abnormalities, striae atrophicae, herniae, dural ectasia, and even neuropsychological problems consequent to biochemical defect. ${ }^{1}$ Clunie and Mason described visceral diverticula in four siblings of a consanguineous marriage, two of whom had marfanoid features. ${ }^{2}$ No similar cases have been published since. We report two siblings with marfanoid phenotype, both of whom developed visceral diverticula and eventration of the diaphragm.

\section{Case histories}

CASE 1 (INDEX CASE)

A 10 year old boy, the second child of consanguineous Sri Lankan parents, had been born after a full term pregnancy and the neonatal period had been uneventful. Development was within normal limits. At 4 years of age mitral valve prolapse was diagnosed at routine examination. At 5.5 years bilateral inguinal herniae were repaired. A few months later he had an episode of bowel obstruction and laparotomy showed volvulus of the stomach. There were no abnormal symptoms referable to the cardiorespiratory system. He did not play active sports. He was found to be myopic at school, and started wearing glasses at 7 years.

General examination showed a prepubertal boy with marfanoid habitus (fig 1 ). His weight was $24 \mathrm{~kg}$ (between 3rd and 10th centiles), height $150.9 \mathrm{~cm}$ ( $>97$ th centile), with a body surface area of $0.97 \mathrm{~m}^{2}$. The lower segment measured $80.5 \mathrm{~cm}$ and arm span was 150.8 $\mathrm{cm}$. He had a long thin face with slightly low set ears. He was highly myopic but without iridodonesis. The fingers were long but Steinberg's sign was negative. Joints were normal without hyperextensibility and there was no pes planus nor cavus. There was no hyperelasticity of the skin. The apex beat was just medial to the midclavicular line with a systolic murmur audible along the left sternal edge. There was decreased air entry in the left lung base posteriorly. Recently he complained of a lump appearing in the right buttock when standing, which was reducible with upward pressure, with an urge to micturate.

Urine nitroprusside test to exclude homocystinuria was negative. Ophthalmological examination showed no evidence of lens dislocation, nor striae angeoides. Intraocular pressure was normal. Uncorrected visual acuity was $1 / 60$ for both eyes. After a 22 dioptres correction, the visual acuity for both eyes was $6 / 18$. Echocardiogram showed moderate mitral valve prolapse and the diameter of the aortic root was $2.1 \mathrm{~cm}$ at the sinus of Valsalva. There was no evidence of aortic dissection nor aortic valve incompetence. An abdominal/pelvic ultrasound revealed a urinary bladder diverticulum and the cystogram confirmed a large diverticulum of the bladder herniating through the pelvic floor into the buttock (fig 2). An initial chest radiograph was normal at 4 years, but at 8 years of age eventration of the left hemidiaphragm was evident, which had progressed to displace the heart to the right, 18 months later (fig 3).

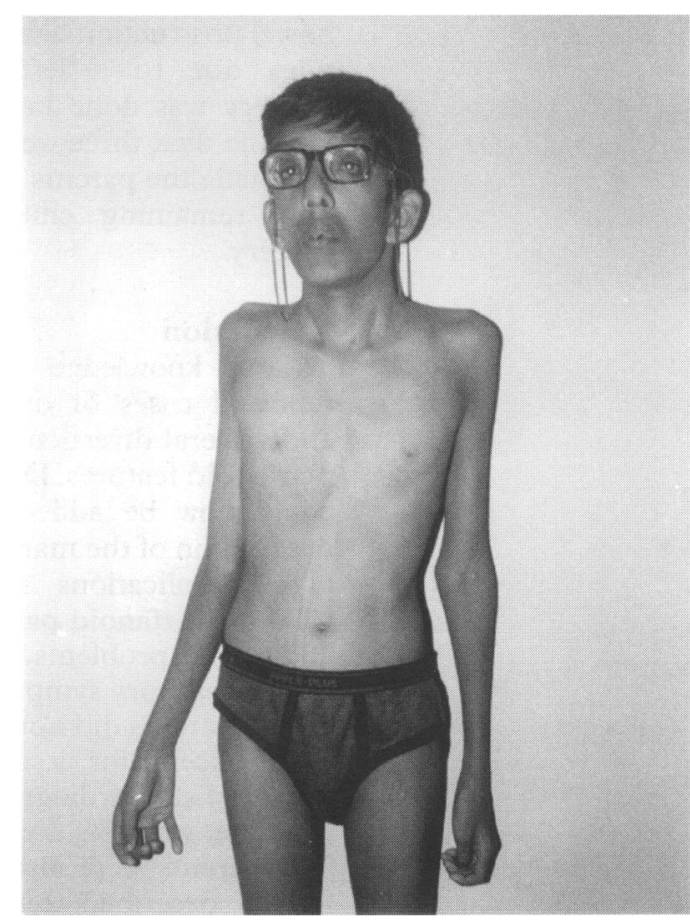

Figure 1 Case 1 showing marfanoid habitus. 


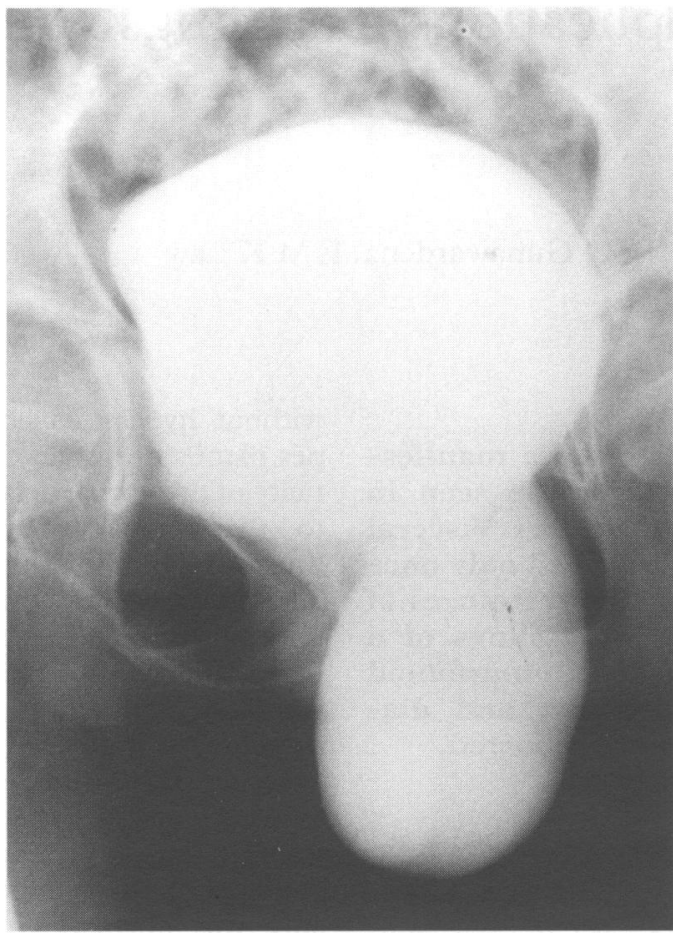

Figure 2 Lateral view of cystogram in case 1 showing the diverticulum herniating through the pelvic floor into the buttock.

CASE 2

Case 2 was the sister of case 1 and was three years older. She also had a marfanoid habitus, had mitral valve prolapse diagnosed at 5 years, and glasses for myopia (without lens dislocation) aged 6 years. Her inguinal herniae were first repaired when she was 2 years old but required two further operations for recurrent hernia. At 8 years she developed bowel obstruction. Malrotation and volvulus (necessitating bowel resection) and multiple colonic diverticula were noted at laparotomy. The next year gangrenous diverticula required further surgery. At 11 years she developed signs of bowel obstruction again, associated with dyspnoea due to a left diaphragmatic hernia. Surgery was done in a hospital in Colombo, and she died three weeks postoperatively. This has made the parents hesitant in allowing their only remaining child to undergo elective surgery.

\section{Discussion}

To our knowledge these are the first two reported cases of diaphragmatic eventration and visceral diverticula in Asian children with marfanoid features. Diaphragmatic eventration could now be added to the still expanding description of the marfanoid phenotype. These two complications should be remembered when a marfanoid patient presents with acute abdominal problems, with or without associated respiratory symptoms.

The parents did not have any marfanoid features except for a systolic murmur in the mother. Echocardiography showed mitral valve prolapse, which is a common isolated finding. The parents were also consanguineous in the family described by Clunie and Mason, ${ }^{2}$

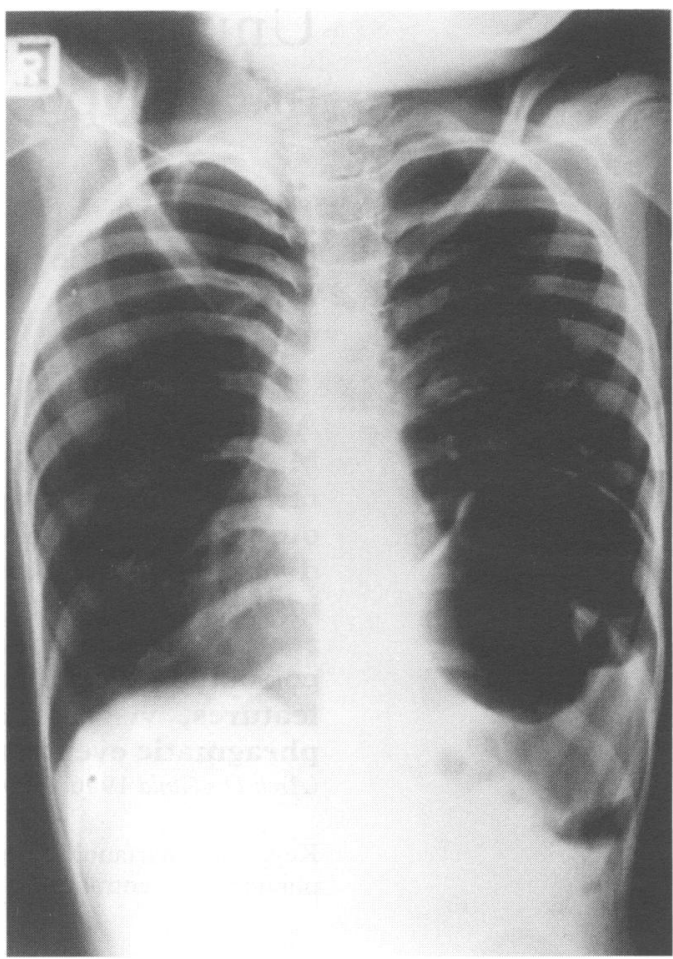

Figure 3 A chest radiograph of case 1 showing the left hemidiaphragmatic eventration with displacement of the heart.

indicating recessive inheritance. Four of these children had visceral diverticula with two of them showing marfanoid features, but not classical Marfan's syndrome. This, along with our family here, may in fact represent a different syndrome with recessive inheritance.

Generalised connective tissue disorder leads to weak diaphragmatic muscles and fibrous tissue, and defective (or even absent) gastrocolic and gastrosplenic ligaments, with consequent inadequate fixation of the stomach. ${ }^{3}$ Similarly, incomplete fixation of the small bowel could account for the malrotation and volvulus in the girl, as in the previous report. ${ }^{2}$

Any defect that causes weakness in the fibromuscular wall of hollow viscera could result in the formation of diverticula spontaneously. It is therefore not surprising that gastrointestinal tract diverticula were described in patients with a Marfan-like disease ${ }^{2}$ and Ehlers-Danlos syndrome. ${ }^{4}$ Urinary bladder diverticulum is a well recognised feature in Ehlers-Danlos syndrome. ${ }^{4}$ In cutis laxa (autosomal recessive) type I, congenital diaphragmatic hernia and diverticula of colon and bladder have been reported; however the prognosis of these patients is poor. ${ }^{5}$ Urinary bladder diverticula have been reported in kinky hair disease ${ }^{6}$ and also in one patient of Clunie and Mason. ${ }^{2}$

1 Pyeritz RE. Marfan syndrome. N Engl f Med 1990; 323: 987-9.

2 Clunie GJA, Mason JM. Visceral diverticula and the Marfan syndrome. Br $\mathcal{F}$ Surg 1962; 50: 51-2.

Cameron AEP, Howard ER. Gastric volvulus in childhood. f Pediatr Surg 1987; 22: 944-7.

4 Schippers E, Dittler HJ. Multiple hollow organ dysplasia in Ehlers-Danlos syndrome. $\mathcal{F}$ Pediatr Surg 1989; 24: 1181-3. 5 Winter RM, Baraitser M. London dysmorphology database (LDDB). Oxford Medical (Computer) Databases. Oxford Oxford University Press, 1993.

6 Menkes JH. Kinky hair disease: twenty five years later. Brain Dev 1988; 10: 77-9. 